OPEN ACCESS

Edited by:

José Bines,

Brazilian National Cancer Institute (INCA), Brazil

Reviewed by:

Marcio Debiasi,

Latin American Cooperative Oncology Group (LACOG), Brazil

Luigi Formisano,

Vanderbilt University Medical Center,

United States

*Correspondence:

Jianlin Lou

jianlinlou@163.com

†These authors have contributed equally to this work

Specialty section:

This article was submitted to

Women's Cancer,

a section of the journal

Frontiers in Oncology

Received: 14 September 2018

Accepted: 01 February 2019

Published: 01 March 2019

Citation:

Yu D, Tong Y, Guo X, Feng L, Jiang Z,

Ying S, Jia J, Fang Y, Yu M, Xia H,

Shi L and Lou J (2019) Diagnostic

Value of Concentration of Circulating

Cell-Free DNA in Breast Cancer: A

Meta-Analysis. Front. Oncol. 9:95.

doi: 10.3389/fonc.2019.00095

\section{Diagnostic Value of Concentration of Circulating Cell-Free DNA in Breast Cancer: A Meta-Analysis}

\author{
Dandan Yu ${ }^{1+}$, Yan Tong ${ }^{2 t}$, Xinnian Guo ${ }^{1}$, Lingfang Feng ${ }^{1}$, Zhaoqiang Jiang ${ }^{1}$, Shibo Ying ${ }^{1}$, \\ Junlin Jia ${ }^{1}$, Yuan Fang ${ }^{1}$, Min Yu ${ }^{1}$, Hailing Xia ${ }^{1}$, Li Shi ${ }^{1}$ and Jianlin Lou ${ }^{1 *}$ \\ ${ }^{1}$ Institute of Occupational Diseases, Zhejiang Academy of Medical Sciences (ZJAMS), Hangzhou, China, ${ }^{2}$ Department of \\ Outpatient Office, Affiliated Hangzhou First People's Hospiital, Zhejiang University School of Medicine, Hangzhou, China
}

The diagnostic value of the concentration of circulating cell-free DNA (cfDNA) for breast cancer has generated inconsistent results. The aim of this study was to evaluate the first diagnostic value of the concentration of cfDNA for breast cancer by meta-analysis. Studies were retrieved by searching PubMed, Cochrane Library, and Web of Science before June 2018. Sensitivity, specificity, diagnostic odds ratio (DOR), the summary receiver operating characteristic (SROC) curve, and the area under curve (AUC) were used to summarize overall diagnostic performance. The random-effects model was used to calculate the pooled statistics. Subgroup analysis and meta-regression analysis were carried out to detect the source of heterogeneity. A total of 13 studies were identified with 1,087 breast cancer patients and 720 healthy controls. Overall, the pooled sensitivity and specificity of concentration of cfDNA for breast cancer were $87 \%(95 \% \mathrm{Cl}, 73-94 \%)$ and 87\% (95\% Cl, 79-93\%), respectively. The pooled DOR was 32.93 (95\% Cl, 13.52-80.19) and the SROC curve revealed an AUC of 0.93 (95\% Cl, 0.91-0.95). Meta-regression analysis showed that no covariate had a significant correlation with relative DOR (RDOR). Publication bias was not detected in this meta-analysis. This meta-analysis indicates that the concentration of cfDNA has potential first diagnostic value for breast cancer and plasma may be a better source of cfDNA for detection of breast cancer.

Keywords: circulating cell-free DNA, concentration, breast cancer, diagnostic value, meta-analysis

\section{INTRODUCTION}

Breast cancer is one of the most common malignancies in women. According to an estimation from the WHO, more than 508,000 women worldwide died from breast cancer in 2011 (1). In China, breast cancer is the fourth most common cancer, with an estimated 150,000 cancer cases in 2012 (2). Early detection and diagnosis of breast cancer has been shown to reduce mortality (3). Currently, mammography is considered the gold standard in breast cancer early detection, which was also proven to be the only screening method to reduce mortality (4). However, mammography may fail to identify patients due to the overlapping dense fibroglandular tissue which reduce the visibility of tumor tissue or even entirely conceal the malignant lesions (5). It was reported that $15-30 \%$ of breast cancer was not detected while using full-field digital mammography (6). In addition, mammography may lead to over-diagnosis and radiation-induced disease. Therefore, in consideration of the limitation of mammography, it is necessary to develop a new non-invasive method to distinguish breast cancer patients from healthy individuals. 
Cells can release DNA into the bloodstream, which is described as circulating cell-free DNA (cfDNA) (7). The cellfree DNA released from tumor cells, in particular, is called circulating tumor DNA (ctDNA) (7). Although cfDNA is also present in healthy individuals, it is significantly increased in cancer subjects (8). cfDNA is recognized as a novel biomarker in the diagnosis of cancer, such as gastric cancer (9), non-small lung cancer (10), and hepatocellular carcinoma (11). There are several detection strategies for breast cancer by cfDNA, such as the concentration of $\mathrm{cfDNA}$, cfDNA integrity, microsatellite alteration, gene mutations, DNA methylation, and so on (12). The concentration of cfDNA is recognized as a quantitative way to detect cfDNA amounts and the initial detection strategy of breast cancer by cfDNA (12). So far, a number of studies have evaluated the diagnostic value of the concentration of cfDNA for breast cancer, but the results are inconsistent. For instance, Agostini et al. (13) reported a high sensitivity of $94.8 \%$ and a high specificity of $100 \%$, while a study by Tang et al. (14) showed a low sensitivity of $65.0 \%$ and a low specificity of $70.0 \%$. Hence, we conducted this meta-analysis to evaluate the first diagnostic value of the concentration of cfDNA for breast cancer.

\section{MATERIALS AND METHODS}

\section{Search Strategy}

The following databases were searched to identify all potentially relevant studies published before June 2018: PubMed, Cochrane Library, and Web of Science. The retrieving query formulation used for the search were ("cell free DNA" OR "circulating DNA"OR "plasma DNA" OR "serum DNA") AND ("breast cancer" OR "breast carcinoma" OR "breast tumor"). Article language was limited to English. All the reference lists of the identified articles and relevant reviews were also manually screened.

\section{Inclusion and Exclusion Criteria}

The inclusion criteria of eligible studies were as follows: (1) studies about the first diagnosis not the recurrent diagnosis of the concentration of cfDNA for breast cancer; (2) studies with sufficient data for describing or calculating the sensitivity and specificity values; and (3) studies that were case-control studies, prospective, and retrospective cohort studies. The exclusion criteria included: (1) studies that were reviews, case-only studies, conference letters, or editorials and (2) studies with duplicate data reported.

\section{Data Extraction and Quality Assessment}

Two reviewers (Dandan Yu and Yan Tong) screened titles, abstracts, and full texts independently according to the above criteria. The following data were extracted from enrolled studies in structured forms: first author's name, publication year, country, sample size, study type, source of cfDNA, time of sample collection, test method, reference gene, cut-off value, sensitivity, and specificity. Subsequently, two reviewers independently evaluated the quality of selected studies according to Quality Assessment of Diagnostic Accuracy Studies-2 (QUADAS-2) (15).

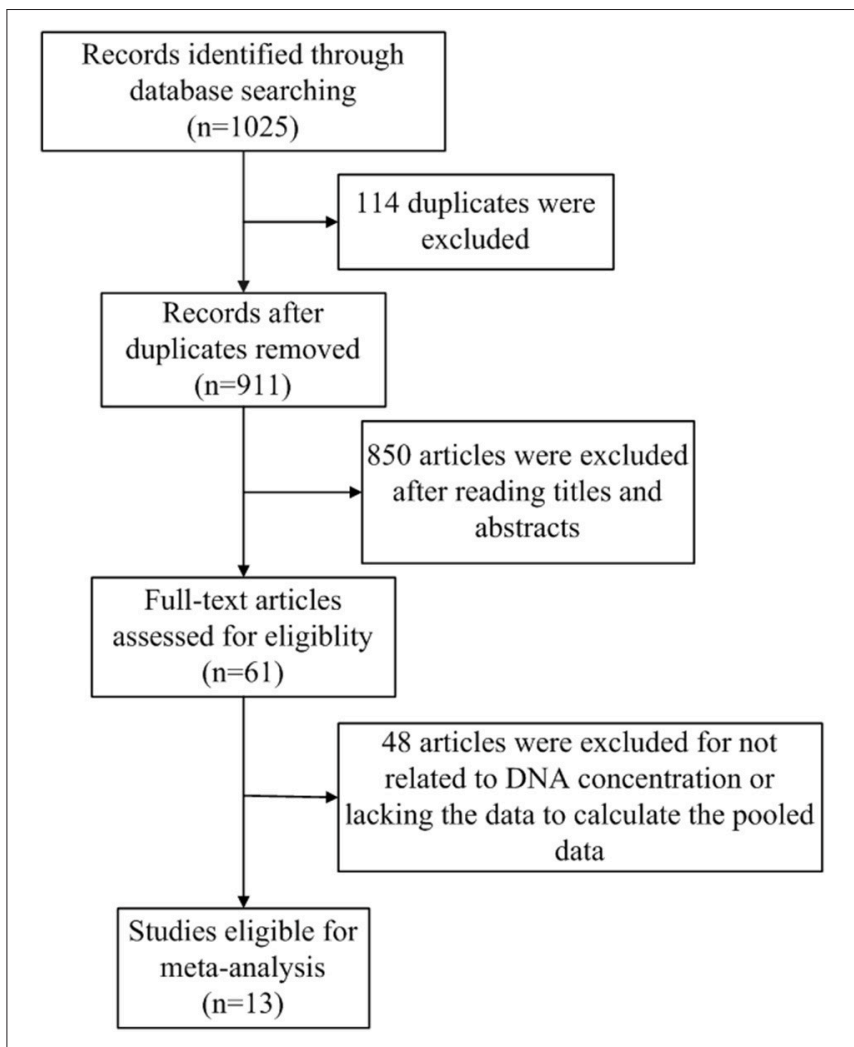

FIGURE 1 | Flow diagram of the study selection process.

\section{Statistical Analysis}

The pooled statistics with $95 \%$ confidence intervals (95\% CIs) of sensitivity, specificity, and diagnostic odds ratio (DOR) were calculated. The summary receiver operating characteristic (SROC) curve and the area under curve (AUC) were used to summarize overall diagnostic performance. Additionally, we used the $\chi^{2}$ test and inconsistency index $\left(I^{2}\right)$ to quantify the statistical heterogeneity between studies. A $P<0.1$ by $\chi^{2}$ test and $I^{2}$ statistic $>50 \%$ indicated substantial heterogeneity (16). A fixed-effects model was used to calculate the pooled statistics if there was no statistical heterogeneity; otherwise, a randomeffects model was conducted. Furthermore, subgroup analysis and meta-regression analysis were carried out to detect the source of heterogeneity. Publication bias was evaluated with Deeks' funnel plot asymmetry test (17). $P<0.05$ indicated potential publication bias.

All statistical analysis was performed with STATA (version 14.0; Stata Corp, College Station, TX). A $P<0.05$ was considered statistically significant, and all $P$-values were two-sided.

\section{RESULTS}

\section{Study Characteristics and Quality Assessment}

The flowchart of literature search and selection were shown in Figure 1. A total of 1,025 records were initially identified by 


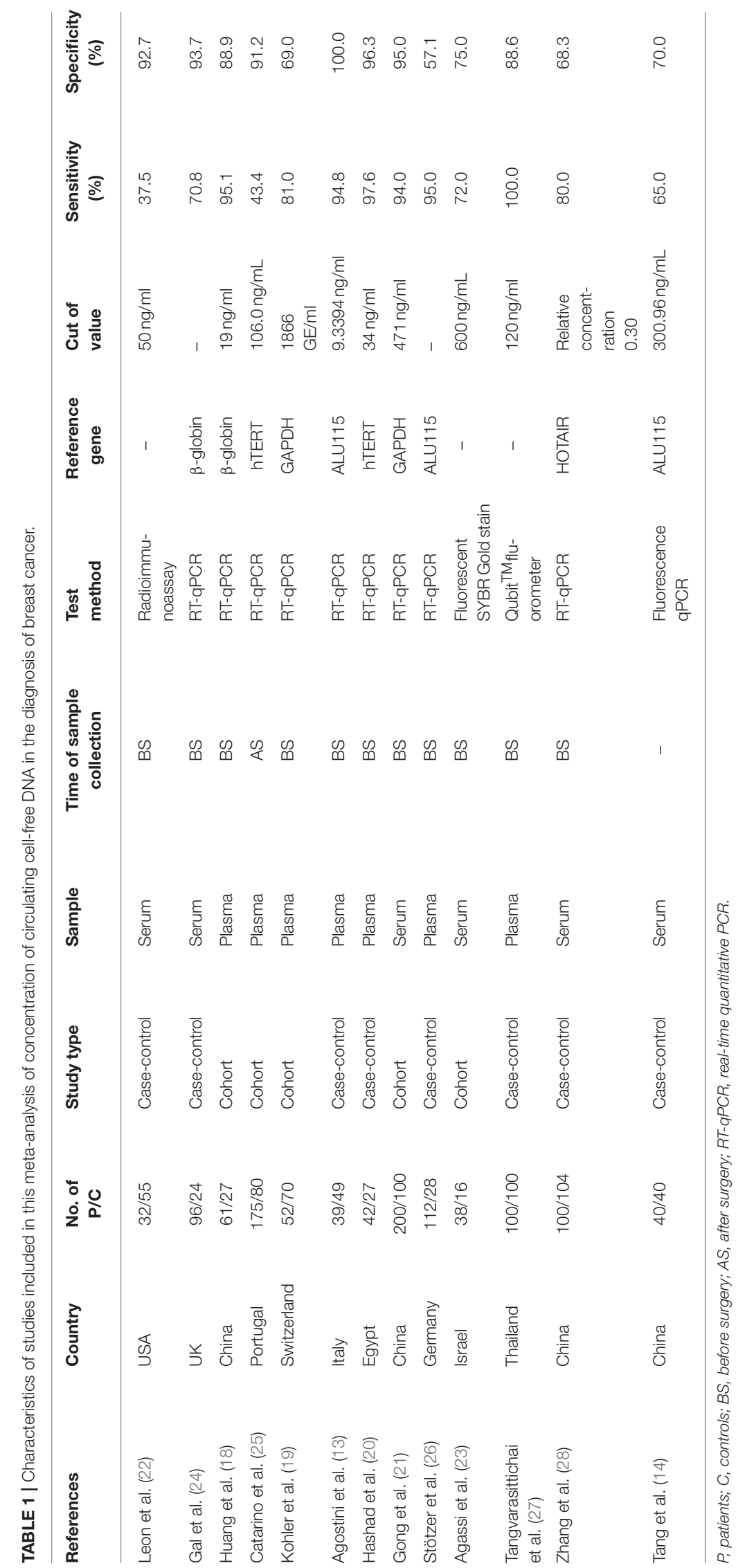


TABLE 2 | Assessment of the methodological quality by QUADAS-2.

\begin{tabular}{|c|c|c|c|c|c|c|c|}
\hline \multirow[t]{2}{*}{ References } & \multicolumn{4}{|c|}{ Risk of bias } & \multicolumn{3}{|c|}{ Applicability concerns } \\
\hline & $\begin{array}{l}\text { Patient } \\
\text { selection }\end{array}$ & $\begin{array}{l}\text { Index } \\
\text { test }\end{array}$ & $\begin{array}{l}\text { Reference } \\
\text { standard }\end{array}$ & $\begin{array}{l}\text { Flow and } \\
\text { timing }\end{array}$ & $\begin{array}{l}\text { Patient } \\
\text { selection }\end{array}$ & $\begin{array}{l}\text { Index } \\
\text { test }\end{array}$ & $\begin{array}{l}\text { Reference } \\
\text { standard }\end{array}$ \\
\hline Leon et al. (22) & (ن) & $\ddot{\circ}$ & (ت) & (ت) & (ت) & (ت) & (ت) \\
\hline Gal et al. (24) & ஜூ) & & $(\bullet)$ & $(\bullet)$ & $\ddot{\circ}$ & $(\bullet)$ & $(\bullet)$ \\
\hline Huang et al. (18) & (ச) & & (ت) & $(\bullet)$ & (ن) & $(\bullet)$ & (ت) \\
\hline Catarino et al. (25 & (ن) & & (ت) & (ت) & (ت) & $(\ddot{)}$ & (ت) \\
\hline Kohler et al. (19) & $(\bullet)$ & & (ت) & (ت) & & $?$ & (ن) \\
\hline Agostini et al. (13) & (ச) & (ت) & (ت) & $(\bullet)$ & (ت) & $(\bullet)$ & (ت) \\
\hline Hashad et al. (20) & (ت) & & (ت) & (ت) & & & (ت) \\
\hline Gong et al. (21) & (ச) & & (ச) & $(\mathcal{)}$ & (ت) & (ச) & (ச) \\
\hline Stötzer et al. (26) & (ச) & & (ت) & $(\ddot{)}$ & & & (ت) \\
\hline Agassi et al. (23) & (ச) & $\ddot{\circ}$ & (ச) & $(\ddot{)}$ & (ت) & $(\ddot{)}$ & (ச) \\
\hline $\begin{array}{l}\text { Tangvarasittichai } \\
\text { et al. (27) }\end{array}$ & & & (ت) & & & & \\
\hline Zhang et al. (28) & $(-)$ & & (ت) & (ت) & (ت) & $(\ddot{)}$ & $(\ddot{)}$ \\
\hline Tang et al. (14) & (ت) & 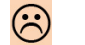 & (ت) & (ت) & (ت) & (ت) & (ت) \\
\hline
\end{tabular}

(-) low risk; $\odot$, nigh rsk; $?$, unclear risk.

our search strategy. Ultimately, 13 studies $(13,14,18-28)$ were eligible for this meta-analysis according to the inclusion criteria and the exclusion criteria. A total of 1,807 subjects including 1,087 breast cancer patients and 720 healthy controls were recruited for analysis. The sensitivity and specificity of cfDNA for breast cancer detection in selected studies ranged from 37.5 to $100 \%$ and from 57.1 to $100 \%$, respectively. Characteristics of these studies are summarized in Table $\mathbf{1 .}$

The quality of selected studies by QUADAS-2 is shown in Table 2. To some extent, most studies had a moderatehigh quality, the overall quality of these included studies were generally robust.

\section{Diagnostic Value of Concentration of cfDNA for Breast Cancer}

The pooled sensitivity and specificity of concentration of cfDNA for breast cancer were $87 \%$ (95\% CI, 73-94\%) and 87\% (95\% CI, 79-93\%), respectively (Figure 2, Table 3 ). The pooled DOR was 32.93 (95\% CI, 13.52-80.19) (Figure 3, Table 3). The SROC curve revealed an AUC of 0.93 (95\% CI, 0.91-0.95) (Figure 4, Table 3).

Sample of cfDNA, the time of sample collection, and test method were taken into consideration for subgroup analysis. With consideration of the sample of cfDNA, studies with cfDNA isolated from plasma revealed that the pooled sensitivity and specificity were $94 \%$ (95\% CI, 80-98\%) and 89\% (95\% CI, 75$96 \%$ ), respectively (Table 3$)$. The pooled DOR was $79.11(95 \%$ CI, 19.13-327.12), and the SROC curve revealed an AUC of 0.97 (95\%CI, 0.95-0.98) (Table 3). Studies with cfDNA isolated from serum revealed that the pooled sensitivity and specificity were 74\% (95\% CI, 56-86\%) and 85\% (95\% CI, 73-92\%), respectively (Table 3). The pooled DOR was 15.80 (95\% CI, 4.51-55.34), and the SROC curve revealed an AUC of 0.87 (95\%CI, 0.84-0.90) (Table 3).

With consideration of the time of sample collection, studies with samples collected before surgery revealed that the pooled sensitivity and specificity were 90\% (95\% CI, 78-96\%) and 88\% (95\% CI, 78-94\%), respectively (Table 3). The pooled DOR was 50.15 (95\% CI, 17.49-143.79), and the SROC curve revealed an AUC of 0.95 (95\% CI, 0.93-0.96) (Table 3).

With consideration of the test method, studies with the test method of real-time qPCR revealed that the pooled sensitivity and specificity were $88 \%$ (95\% CI, 77-95\%) and 89\% (95\% CI, 77-95\%), respectively (Table 3 ). The pooled DOR was 46.29 (95\% CI, 15.82-135.45), and the SROC curve revealed an AUC of 0.95 (95\% CI, 0.93-0.96) (Table 3). Studies without the test method of real-time qPCR showed that the pooled sensitivity and specificity were $85 \%$ (95\% CI, 29-99\%) and 84\% (95\% CI, 73$91 \%$ ), respectively (Table 3 ). The pooled DOR was 16.60 (95\% CI, 2.62-105.31), and the SROC curve revealed an AUC of 0.88 (95\% CI, 0.85-0.91) (Table 3).

\section{Meta-Regression Analysis and Publication Bias}

To reveal the sources of heterogeneity, we conducted a meta-regression analysis. Covariates including "publication year (recent 5 years)," "region (Asian)," “sample (plasma)," "time 


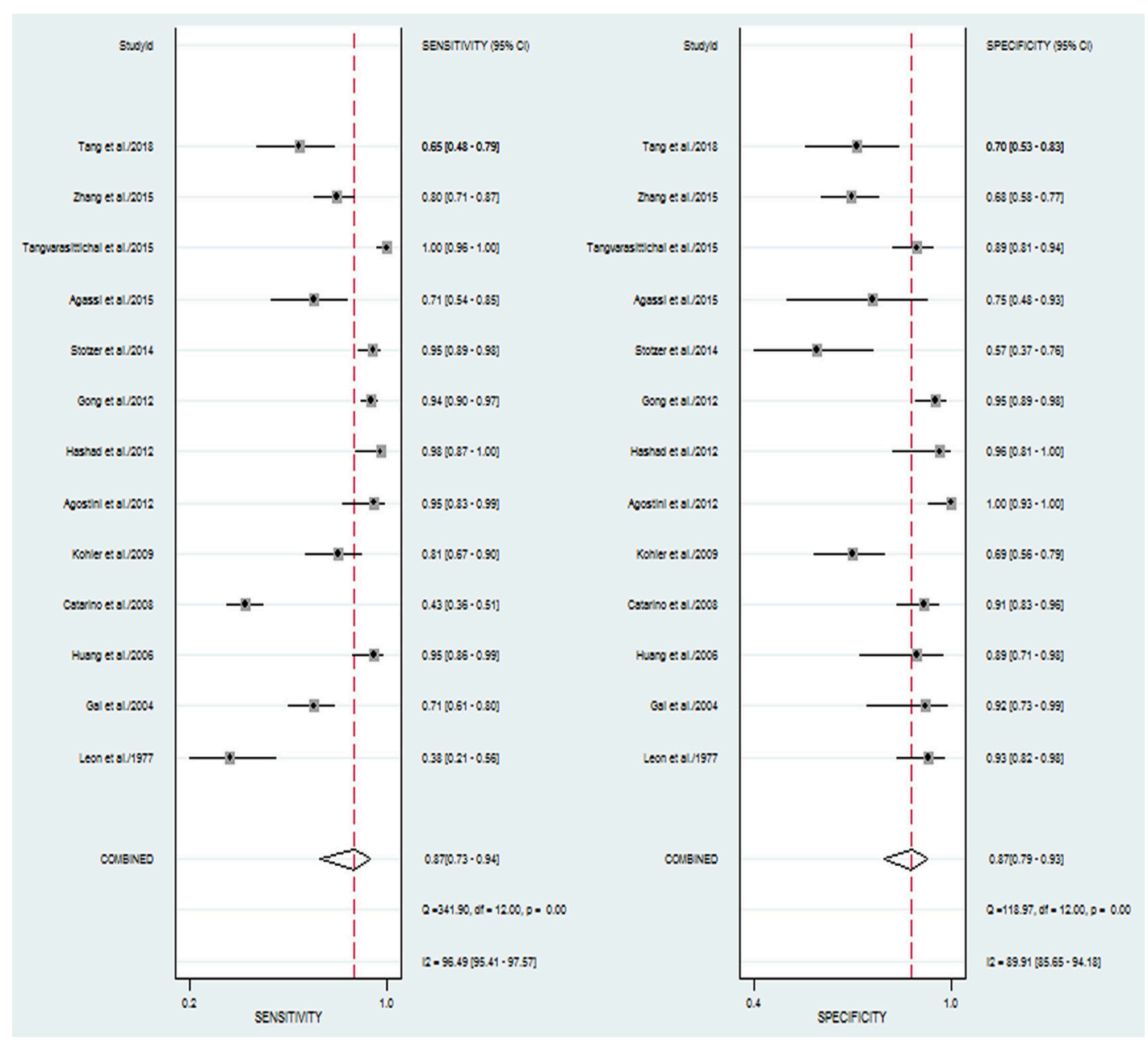

FIGURE 2 | Forest plots of sensitivity and specificity for diagnostic value of concentration of circulating cell-free DNA for breast cancer.

TABLE 3 | Summary of main results of diagnostic value of concentration of cfDNA for breast cancer.

\begin{tabular}{llcccccccc}
\hline Study group & & $\boldsymbol{N}$ & Sensitivity (95\% Cl) & $\boldsymbol{I}^{\mathbf{2}}$ (\%) & Specificity (95\% Cl) & $\boldsymbol{I}^{\mathbf{2}}$ (\%) & DOR (95\% Cl) & $\boldsymbol{I}^{\mathbf{2}}(\mathbf{\%})$ & $\mathbf{A U C}(\mathbf{9 5 \%} \mathbf{C l})$ \\
\hline All & & 13 & $87 \%(73 \%-94 \%)$ & 96.49 & $87 \%(79 \%-93 \%)$ & 89.91 & $32.93(13.52-80.19)$ & 85.8 & $0.93(0.91-0.95)$ \\
Sample of cfDNA & plasma & 7 & $94 \%(80 \%-98 \%)$ & 98.52 & $89 \%(75 \%-96 \%)$ & 94.26 & $79.11(19.13-327.12)$ & 85.0 & $0.97(0.95-0.98)$ \\
& serum & 6 & $74 \%(56 \%-86 \%)$ & 92.54 & $85 \%(73 \%-92 \%)$ & 85.77 & $15.80(4.51-55.34)$ & 88.3 & $0.87(0.84-0.90)$ \\
Time of sample collection & before surgery & 11 & $90 \%(78 \%-96 \%)$ & 94.1 & $88 \%(78 \%-94 \%)$ & 89.17 & $50.15(17.49-143.79)$ & 86.1 & $0.95(0.93-0.96)$ \\
Test method & RT-qPCR & 9 & $88 \%(77 \%-95 \%)$ & 97.52 & $89 \%(77 \%-95 \%)$ & 93.66 & $46.29(15.82-135.45)$ & 86.8 & $0.95(0.93-0.96)$ \\
& Not RT-qPCR & 4 & $85 \%(29 \%-99 \%)$ & 95.04 & $84 \%(73 \%-91 \%)$ & 74.64 & $16.60(2.62-105.31)$ & 85.0 & $0.88(0.85-0.91)$
\end{tabular}

$N$, number of studies.

of sample collection (before surgery)," and "method (real-time quantitative PCR)" were assessed. We used these covariates to assess their effects on the RDOR. None of them showed any definite influence on heterogeneity and the details of the calculation were showed in Table 4. Deeks' funnel plot asymmetry test was used to assess publication bias. The $P$-value was 0.65 , which showed there was no significant evidence of publication bias (Figure 5).

\section{DISCUSSION}

As is known, mammography as a screening method for breast cancer has the limitation of having low sensitivity. Hence, the circulating biomarkers, which can be easily collected and measured, have attracted the attention of more researchers. A correlation between increased levels of cfDNA and cancer has been widely studied $(29,30)$. Nowadays, it has been widely 


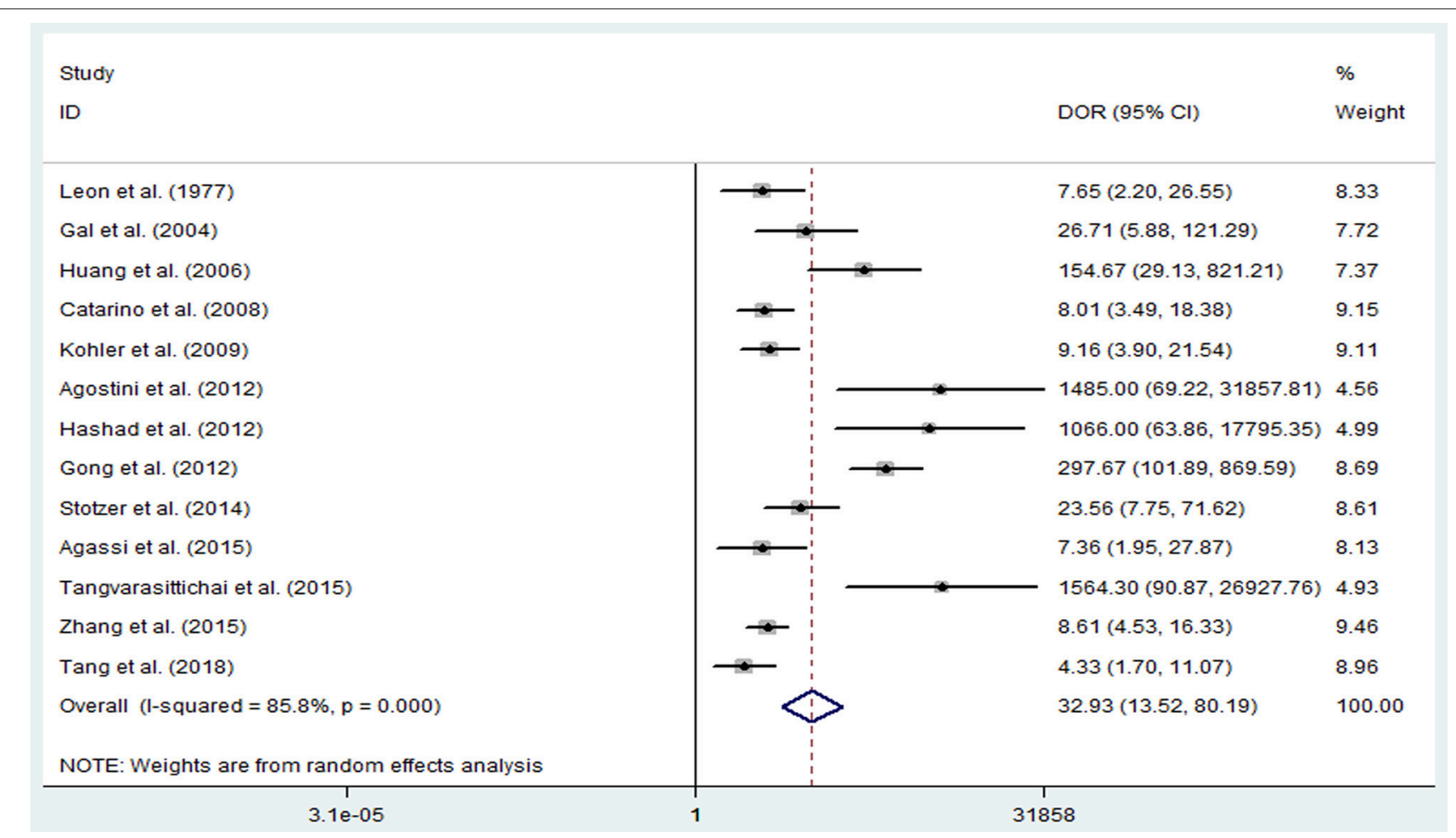

FIGURE 3 | Diagnostic odds ratio of sensitivity and specificity for diagnostic value of concentration of circulating cell-free DNA for breast cancer.

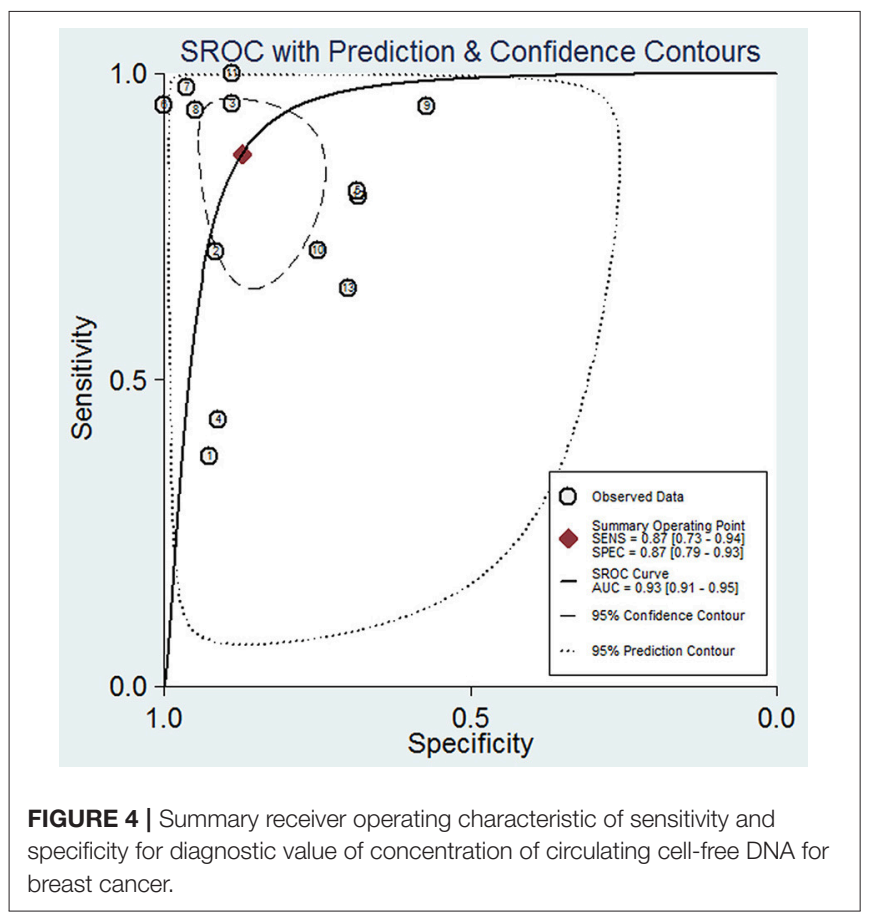

accepted that a solid tumor will obviously increase cfDNA concentrations (31). It was also demonstrated that the circulating cfDNA concentrations of breast cancer patients were significantly higher than healthy controls (25).
TABLE 4 | Meta-regression of effects of study characteristics on diagnostic accuracy of concentration of cfDNA.

\begin{tabular}{lcccc}
\hline Covariates & Coefficient & SE & $\boldsymbol{P}$ & RDOR (95\% Cl) \\
\hline Year & 1.526 & 6.252 & 0.299 & $4.598(0.184-114.463)$ \\
Region & -1.862 & 0.207 & 0.206 & $0.155(0.007-3.662)$ \\
Sample & -2.179 & 0.142 & 0.127 & $0.113(0.006-2.212)$ \\
Time of sample collection & -2.133 & 0.171 & 0.182 & $0.118(0.004-3.576)$ \\
Test method & 0.283 & 1.888 & 0.848 & $1.328(0.046-38.349)$
\end{tabular}

SE, standard error.

During the past decades, biomarkers of breast cancer have been widely discovered, such as CEA, CA15-3, HER2, and so on. CEA, which exists in the breast ductal secretions, showed a lower sensitivity of $58 \%$, a lower AUC of 0.8750 , and a lower DOR of 7.70 for breast cancer diagnosis (32). One study combined four biomarkers, including M-CSF, MMP-9, TIMP-1, and CA 15-3, drew a lower sensitivity, specificity, and AUC for breast cancer. The sensitivity, specificity, and AUC were $84 \%, 83 \%$, and 0.9125 , respectively (33).

In this study, we found a high diagnostic value of concentration of cfDNA, of which the sensitivity and specificity reached 87 and $87 \%$, respectively. Additionally, AUC is considered as an indicator of good diagnostic performance when the value is $>0.90$ (34). Therefore, the value of 0.93 in our study indicated that concentration of cfDNA had a good diagnostic accuracy for breast cancer. According to 


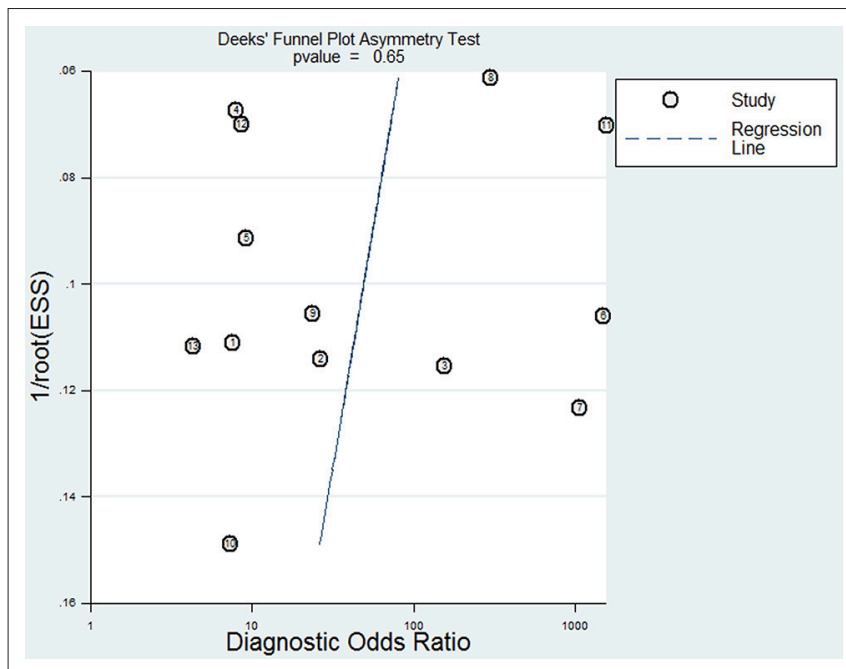

FIGURE 5 | Deeks' funnel plot for the assessment of potential publication bias in this meta-analysis.

the above results, we can see that compared with other biomarkers of breast cancer, whether they are novel or classical, cfDNA may have better performance on detection of breast cancer.

We also conducted subgroup analyses. The results showed there were no obvious differences among the subgroup analyses for the sensitivity, specificity, and AUC. But the DORs varied from 15.80 to 79.11 . When we only incorporated the studies using plasma samples, the highest sensitivity, specificity, and AUC of cfDNA on breast cancer detection were obtained, and they were also higher than pooled sensitivity, specificity, and AUC, respectively. These results indicated that plasma may be a better source for cfDNA in detection of breast cancer.

The original studies included in our analysis showed obvious differences in sensitivity and specificity. It is widely accepted that malignancy will increase the bloodstream concentration of cfDNA. However, in some abnormal condition, such as inflammation (35), arthritis (36), trauma (37), and even after exhaustive exercise (38), the concentration of cfDNA increases too. It reminds us that benign breast lesions and other illness should be firstly excluded when we use cfDNA to detect breast cancer. Also, the diagnostic efficiency of cfDNA on breast cancer detection can be much improved in combination with other known biomarkers (CA15-3, CEA, HER2, and so on) for breast cancer.

This study also contains several limitations. Firstly, as only 13 studies were included in this meta-analysis, compounded by

\section{REFERENCES}

1. World Health Organization. Breast Cancer: Prevention and Control (2013). Available online at: http://www.who.int/cancer/detection/breastcancer/en/ index1.html the small number of included studies, it may be insufficient to yield a robust result. Secondly, heterogeneity existed between the selected studies, although it was impossible to determine all sources of heterogeneity. We did not include some covariates due to the unavailable data. These probable covariates included tumor stage, metastasis condition, tumor size, the risk of breast cancer, and so on. Thirdly, subgroup analyses on several covariates were unable to perform due to the various data, such as reference gene and cut-off value. Moreover, language bias might exist due to the references being restricted to English. Even so, to our knowledge, this study is the first to systematically evaluate the diagnostic value of the concentration of cfDNA in breast cancer in the whole population and various subgroups.

This meta-analysis suggests that the concentration of cfDNA has potential first diagnostic value for breast cancer due to the high sensitivity, specificity and AUC, and it may be a potential screening tool for breast cancer. In particular, plasma may be a better source of cfDNA in detection of breast cancer. Further large-scaled, well-designed studies are required to confirm our findings, and to provide a basis for future clinical practice. Moreover, the first diagnostic value of concentration of cfDNA for breast cancer could be validated by clinical trials.

\section{DATA AVAILABILITY}

All datasets generated for this study are included in the manuscript.

\section{AUTHOR CONTRIBUTIONS}

DY and YT made substantial contributions to conception and design, acquisition of data, analysis of data, and drafting the manuscript. XG was involved in design and drafting the manuscript. LF, ZJ, and SY were involved in drafting the manuscript and statistical analysis. JJ and YF provided support in statistical analysis. MY and HX were involved in data interpretation. LS participated in the preparation of the manuscript and provided general support. JL designed, coordinated, and supervised the study and he was involved in data analysis and interpretation and drafted the manuscript. All authors read and approved the final manuscript.

\section{FUNDING}

This work was supported by grants from the Zhejiang Committee of Science and Technology (2014C03030), Zhejiang Provincial Program for the Cultivation of High-Level Innovative Health Talents (2014). 
specific biomarker profile. Oncol Rep. (2011) 26:1051-6. doi: 10.3892/or. 2011.1420

4. Lacombe J, Mange A, Solassol J. Use of autoantibodies to detect the onset of breast cancer. J Immunol Res. (2014) 2014:574981. doi: 10.1155/2014/574981

5. Gilbert FJ, Tucker L, Young KC. Digital breast tomosynthesis (DBT): a review of the evidence for use as a screening tool. Clin Radiol. (2016) 71:141-50. doi: 10.1016/j.crad.2015.11.008

6. Duncan KA, Needham G, Gilbert FJ, Deans HE. Incident round cancers: what lessons can we learn? Clin Radiol. (1998) 53:29-32. doi: 10.1016/S0009-9260(98)80030-5

7. Volckmar AL, Sultmann H, Riediger A, Fioretos T, Schirmacher P, Endris $\mathrm{V}$, et al. A field guide for cancer diagnostics using cell-free DNA: from principles to practice and clinical applications. Genes Chromosomes Cancer. (2018) 57:123-39. doi: 10.1002/gcc.22517

8. Mouliere F, El Messaoudi S, Pang D, Dritschilo A, Thierry AR. Multimarker analysis of circulating cell-free DNA toward personalized medicine for colorectal cancer. Mol Oncol. (2014) 8:927-41. doi: 10.1016/j.molonc.2014.02.005

9. Gao Y, Zhang K, Xi H, Cai A, Wu X, Cui J, et al. Diagnostic and prognostic value of circulating tumor DNA in gastric cancer: a meta-analysis. Oncotarget. (2017) 8:6330-40. doi: 10.18632/oncotarget.14064

10. Jiang T, Zhai C, Su C, Ren S, Zhou C. The diagnostic value of circulating cell free DNA quantification in non-small cell lung cancer: a systematic review with meta-analysis. Lung Cancer. (2016) 100:63-70. doi: 10.1016/j.lungcan.2016.06.013

11. Liao W, Mao Y, Ge P, Yang H, Xu H, Lu X, et al. Value of quantitative and qualitative analyses of circulating cell-free DNA as diagnostic tools for hepatocellular carcinoma: a meta-analysis. Medicine. (2015) 94:e722. doi: 10.1097/MD.0000000000000722

12. Wang R, Li X, Zhang H, Wang K, He J. Cell-free circulating tumor DNA analysis for breast cancer and its clinical utilization as a biomarker. Oncotarget. (2017) 8:75742-55. doi: 10.18632/oncotarget.20608

13. Agostini M, Enzo MV, Bedin C, Belardinelli V, Goldin E, Del Bianco P, et al. Circulating cell-free DNA: a promising marker of regional lymphonode metastasis in breast cancer patients. Cancer Biomark. (2012) 11:89-98. doi: 10.3233/CBM-2012-0263

14. Tang Z, Li L, Shen L, Shen X, Ju S, Cong H. Diagnostic value of serum concentration and integrity of circulating cell-free DNA in breast cancer: a comparative study with CEA and CA15-3. Lab Med. (2018) 49:323-8. doi: 10.1093/labmed/lmy019

15. Whiting PF, Rutjes AW, Westwood ME, Mallett S, Deeks JJ, Reitsma JB, et al. QUADAS-2: a revised tool for the quality assessment of diagnostic accuracy studies. Ann Intern Med. (2011) 155:529-36. doi: 10.7326/0003-4819-155-8-201110180-00009

16. Higgins JP, Thompson SG, Deeks JJ, Altman DG. Measuring inconsistency in meta-analyses. BMJ. (2003) 327:557-60. doi: 10.1136/bmj.327.7414.557

17. Deeks JJ, Macaskill P, Irwig L. The performance of tests of publication bias and other sample size effects in systematic reviews of diagnostic test accuracy was assessed. J Clin Epidemiol. (2005) 58:882-93. doi: 10.1016/j.jclinepi.2005.01.016

18. Huang $\mathrm{ZH}$, Li LH, Hua D. Quantitative analysis of plasma circulating DNA at diagnosis and during follow-up of breast cancer patients. Cancer Lett. (2006) 243:64-70. doi: 10.1016/j.canlet.2005.11.027

19. Kohler C, Radpour R, Barekati Z, Asadollahi R, Bitzer J, Wight E, et al. Levels of plasma circulating cell free nuclear and mitochondrial DNA as potential biomarkers for breast tumors. Mol Cancer. (2009) 8:105. doi: 10.1186/1476-4598-8-105

20. Hashad D, Sorour A, Ghazal A, Talaat I. Free circulating tumor DNA as a diagnostic marker for breast cancer. J Clin Lab Anal. (2012) 26:467-72. doi: $10.1002 /$ jcla.21548

21. Gong B, Xue J, Yu J, Li H, Hu H, Yen H, et al. Cell-free DNA in blood is a potential diagnostic biomarker of breast cancer. Oncol Lett. (2012) 3:897-900. doi: $10.3892 / 01.2012 .576$

22. Leon SA, Shapiro B, Sklaroff DM, Yaros MJ. Free DNA in the serum of cancer patients and the effect of therapy. Cancer Res. (1977) 37:646-50.
23. Agassi R, Czeiger D, Shaked G, Avriel A, Sheynin J, Lavrenkov K, et al. Measurement of circulating cell-free DNA levels by a simple fluorescent test in patients with breast cancer. Am J Clin Pathol. (2015) 143:18-24. doi: 10.1309/AJCPI5YHG0OGFAHM

24. Gal S, Fidler C, Lo YM, Taylor M, Han C, Moore J, et al. Quantitation of circulating DNA in the serum of breast cancer patients by real-time PCR. $\mathrm{BrJ}$ Cancer. (2004) 90:1211-5. doi: 10.1038/sj.bjc.6601609

25. Catarino R, Ferreira MM, Rodrigues H, Coelho A, Nogal A, Sousa A, et al. Quantification of free circulating tumor DNA as a diagnostic marker for breast cancer. DNA Cell Biol. (2008) 27:415-21. doi: 10.1089/dna. 2008.0744

26. Stotzer OJ, Lehner J, Fersching-Gierlich D, Nagel D, Holdenrieder S. Diagnostic relevance of plasma DNA and DNA integrity for breast cancer. Tumour Biol. (2014) 35:1183-91. doi: 10.1007/s13277-013-1158-4

27. Tangvarasittichai $\mathrm{O}$, Jaiwang $\mathrm{W}$, Tangvarasittichai $\mathrm{S}$. The plasma DNA concentration as a potential breast cancer screening marker. Indian J Clin Biochem. (2015) 30:55-8. doi: 10.1007/s12291-013-0407-Z

28. Zhang L, Song X, Wang X, Xie Y, Wang Z, Xu Y, et al. Circulating DNA of HOTAIR in serum is a novel biomarker for breast cancer. Breast Cancer Res Treat. (2015) 152:199-208. doi: 10.1007/s10549-015-3431-2

29. Gordevicius J, Krisciunas A. Cell-free DNA modification dynamics in abiraterone acetate-treated prostate cancer patients. Clin Cancer Res. (2018) 24:3317-24. doi: 10.1158/1078-0432.CCR-18-0101

30. Park YR, Kim YM, Lee SW, Lee HY, Lee GE, Lee JE, et al. Optimization to detect TP53 mutations in circulating cell-free tumor DNA from patients with serous epithelial ovarian cancer. Obstet Gynecol Sci. (2018) 61:328-36. doi: 10.5468/ogs.2018.61.3.328

31. Ravelli A, Reuben JM, Lanza F, Anfossi S, Cappelletti MR, Zanotti L, et al. Breast cancer circulating biomarkers: advantages, drawbacks, and new insights. Tumour Biol. (2015) 36:6653-65. doi: 10.1007/s13277-0 15-3944-7

32. Tang S, Zhou F, Sun Y, Wei L, Zhu S, Yang R, et al. CEA in breast ductal secretions as a promising biomarker for the diagnosis of breast cancer: a systematic review and meta-analysis. Breast Cancer. (2016) 23:813-9. doi: 10.1007/s12282-016-0680-9

33. Lawicki S, Glazewska EK, Sobolewska M, Bedkowska GE, Szmitkowski M. Plasma levels and diagnostic utility of macrophage colony-stimulating factor, matrix metalloproteinase-9, and tissue inhibitor of metalloproteinases1 as new biomarkers of breast cancer. Ann Lab Med. (2016) 36:223-9. doi: 10.3343/alm.2016.36.3.223

34. Swets JA. Measuring the accuracy of diagnostic systems. Science. (1988) 240:1285-93. doi: 10.1126/science.3287615

35. Herrera CA, Stoerker J, Carlquist J, Stoddard GJ, Jackson M, Esplin S, et al. Cell-free DNA, inflammation, and the initiation of spontaneous term labor. Am J Obstet Gynecol. (2017) 217:583.e1-8. doi: 10.1016/j.ajog.2017.05.027

36. Leon SA, Revach M, Ehrlich GE, Adler R, Petersen V, Shapiro B. DNA in synovial fluid and the circulation of patients with arthritis. Arthritis Rheum. (1981) 24:1142-50. doi: 10.1002/art.1780240905

37. Lo YM, Rainer TH, Chan LY, Hjelm NM, Cocks RA. Plasma DNA as a prognostic marker in trauma patients. Clin Chem. (2000) 46:319-23.

38. Atamaniuk J, Vidotto C, Tschan H, Bachl N, Stuhlmeier KM, Muller MM. Increased concentrations of cell-free plasma DNA after exhaustive exercise. Clin Chem. (2004) 50:1668-70. doi: 10.1373/clinchem.2004.034553

Conflict of Interest Statement: The authors declare that the research was conducted in the absence of any commercial or financial relationships that could be construed as a potential conflict of interest.

Copyright $\odot 2019$ Yu, Tong, Guo, Feng, Jiang, Ying, Jia, Fang, Yu, Xia, Shi and Lou. This is an open-access article distributed under the terms of the Creative Commons Attribution License (CC BY). The use, distribution or reproduction in other forums is permitted, provided the original author(s) and the copyright owner(s) are credited and that the original publication in this journal is cited, in accordance with accepted academic practice. No use, distribution or reproduction is permitted which does not comply with these terms. 